\title{
Prevalencia de variantes anatómicas naso-sinusales: Importancia en el informe radiológico y en la cirugía endoscópica funcional
}

\author{
Dres. José A. de Grazia $K^{(1)}$, Gonzalo Miranda $G^{(2)}$, Katherine Walker ${ }^{(3)}$, Sebastián Aguirre $V^{(4)}$.
}

1. Médico Residente de Radiología, Centro de Imagenología Hospital Clínico Universidad de Chile. Santiago - Chile.

2. Médico Neurorradiólogo, Centro de Imagenología Hospital Clínico Universidad de Chile. Santiago - Chile.

3. Médico Otorrinolaringólogo, Servicio de Otorrinolaringología Hospital Clínico Universidad de Chile. Santiago - Chile.

4. Médico Cirujano, Facultad de Medicina Universidad de Chile. Santiago - Chile.

Prevalence of sino-nasal anatomical variations: Importance in the radiological report and functional endoscopic surgery

\begin{abstract}
Objective: To describe the prevalence in computed tomography (CT) of the main anatomical variations of the sino-nasal region, in a sample of the Chilean population, and to emphasize the importance of reporting them in the radiology report. Materials and methods: 100 paranasal sinus CT performed at our hospital were retrospectively evaluated. Each CT was evaluated by a neuro-radiologist and a third-year Radiology resident, recording the main anatomical variations of the structure and bone pneumatization of this region. Patients younger than 18 yrs of age, and patients with significant distortion of the sino-nasal anatomy (e.g. tumors, history of facial fracture), were excluded. Results: The different anatomical variations were found in frequencies similar to those described in the international literature. The most common anatomical variation was deviated septum (83\%), followed by the presence of Agger nasi cell (66\%) and septal spur (45\%). The upper attachment of the uncinate process was $68 \%$ in the lamina papyracea, $24 \%$ in the cribriform plate and $8 \%$ in the middle turbinate. Configuration of the ethmoid roof, according to the Keros classification, was $2 \%$ type I, $28 \%$ type II and $70 \%$ type III. The frequency of Keros type III ethmoid configuration was higher than that described in classical studies. The degree of sphenoid sinus pneumatization, according to the Hamberger classification, was $80 \%$ sellar, $19 \%$ presellar and $1 \%$ conchal. In 5\%, dehiscence of the bone wall in relation to the carotid channel was recorded, one of these being bilateral. Conclusion: The study of paranasal sinus CT is useful to characterize the anatomical variations of the sino-nasal region. A precise anatomical knowledge of this region is important; variations in the radiology report should be noted, especially if there is a plan to perform functional endoscopic surgery. Keywords: Anatomical variantions, Computed tomography, Sinuses.
\end{abstract}

Resumen: Objetivo: Describir la prevalencia en tomografía computada (TC) de las principales variantes anatómicas de la región naso-sinusal en una muestra de población chilena y destacar la importancia de reportarlas en el informe radiológico. Materiales y método: Se evaluaron retrospectivamente 100 TC de cavidades paranasales realizadas en nuestro hospital. Cada una de las TC fue evaluada por un neurorradiólogo y un residente de tercer año de Radiología, registrando las principales variantes anatómicas de la estructura y neumatización óseas de esta región. Se excluyó a pacientes menores de 18 años y a pacientes que presentaban distorsión significativa de la anatomía naso-sinusal (ej: tumores, antecedente de fractura facial). Resultados: Se encontraron las distintas variantes anatómicas en frecuencias similares a las descritas en la literatura internacional. $\mathrm{La}$ variante anatómica más común fue la desviación del tabique (83\%), seguida de la presencia de celdilla Agger Nasi (66\%) y espolón del tabique (45\%). La inserción superior del proceso uncinado fue $68 \%$ en la lámina papirácea, $24 \%$ en la lámina cribosa y $8 \%$ en el cornete medio. La configuración del techo etmoidal, según la clasificación de Keros, fue 2\% tipo I, 28\% tipo II y 70\% tipo III. La frecuencia de configuración etmoidal tipo III de Keros fue mayor que la descrita en estudios clásicos. El grado de neumatización del seno esfenoidal, según la clasificación de Hamberger, fue $80 \%$ selar, $19 \%$ preselar y $1 \%$ conchal. En $5 \%$ se registró dehiscencia de la pared ósea en relación al canal carotídeo, siendo uno de estos bilateral.

Conclusión: El estudio con TC de cavidades paranasales resulta útil para caracterizar las variantes anatómicas de la región naso-sinusal. Es importante un conocimiento anatómico preciso de esta región, debiendo reportarse las variantes en el informe radiológico, en especial si se planea realizar cirugía endoscópica funcional. Palabras clave: Senos paranasales, Tomografía computada, Variantes anatómicas. 
de Grazia J, et al. Prevalencia de variantes anatómicas naso-sinusales: Importancia en el informe radiológico y en la cirugía endoscópica funcional. Rev Chil Radiol 2014; 20(1): 5-12.

Correspondencia: Dr. José A. de Grazia K. / ja.degrazia@gmail.com

Trabajo recibido el 22 de marzo de 2013. Aceptado para publicación el 12 de febrero de 2014.

\section{Introducción}

Las fosas nasales y los senos paranasales poseen una anatomía compleja y en su conjunto constituyen una unidad funcional. Las anomalías congénitas y las variantes anatómicas normales de esta región son importantes, ya sea por sus consecuencias patológicas o porque pueden constituir una dificultad técnica durante la cirugía ${ }^{(1)}$. Es importante un conocimiento preciso de las variantes anatómicas naso-sinusales del paciente si se planea realizar una cirugía endoscópica funcional (CEF) de senos paranasales $^{(2)}$.

Los métodos de imagen disponibles en la actualidad han contribuido en forma sustancial al entendimiento de la anatomía y patología de los senos paranasales. La radiografía convencional ya no es suficiente para una adecuada evaluación de las variantes anatómicas ni de la patología nasosinusal ${ }^{(3)}$. La tomografía computada (TC) ha surgido como el método de elección para el estudio por imágenes de los senos paranasales, pues permite una detallada caracterización de la arquitectura naso-sinusal y de las enfermedades que afectan a esta región, con una alta resolución espacial ${ }^{(3,4)}$. La resonancia magnética (RM) proporciona buena resolución de contraste, que permite evaluar masas de tejido blando y la extensión de enfermedades infecciosas y neoplásicas con gran detalle ${ }^{(3,4)}$, pero es inferior que la TC para la evaluación de las estructuras óseas.

El presente estudio describe la prevalencia en TC de las principales variantes anatómicas de la región naso-sinusal en una muestra de población chilena.

\section{Materiales y método}

Se evaluaron en forma retrospectiva $100 \mathrm{TC}$ de cavidades paranasales realizadas durante el primer semestre del año 2011 en el Centro de Imagenología de nuestro hospital. Los exámenes fueron realizados en un tomógrafo SOMATON Sensation $₫$ (Siemens $®$ ) de 64 canales, con el cual se hizo adquisición volumétrica, tras lo cual se realizaron reconstrucciones axiales cada $5 \mathrm{~mm}$ y reconstrucciones coronales y sagitales cada $3 \mathrm{~mm}$, todas en ventana ósea. No se utilizó medio de contraste endovenoso. Se excluyó del estudio a pacientes menores de 18 años de edad y a pacientes que presentaban distorsión significativa de la anatomía naso-sinusal (tumores, mucormicosis, antecedente de fractura facial, postoperados de CEF). Cada una de las TC fue evaluada por un neurorradiólogo y un residente de tercer año de Radiología en forma ciega uno del otro. Los casos en los cuales existió discordancia interobservador fueron evaluados en conjunto, para obtener un consenso. Se registraron ${ }^{(5,6)}$ :

- Proceso uncinado: zona de inserción superior y presencia de neumatización (bulla uncinada).

- Cornetes: configuración y presencia de neumatización del cornete medio, presencia de cornete medio secundario y presencia de concha suprema.

- Seno frontal: presencia de hipoplasia o agenesia, lugar de drenaje y clasificación de Kuhn (tipo 10 pequeña celdilla sobre Agger Nasi, tipo 2 o varias celdillas sobre Agger Nasi, tipo 3 o gran celda sobre Agger Nasi con extensión al seno frontal y tipo 4 o celdilla aislada en el seno frontal)

- Celdillas etmoidales: número de celdillas, presencia de eminencia nasal neumatizada (Agger Nasi), presencia de celdilla infraorbitaria (Haller) y presencia de celdilla esfenoetmoidal (Onodi).

- Seno maxilar: presencia de hipoplasia o agenesia.

- Seno esfenoidal: presencia de tabique intraesfenoidal (número e inserción), dehiscencia de pared ósea en relación al canal carotídeo y clasificación de Hamberger para el grado de neumatización (selar, preselar o conchal).

- Fosa olfatoria: clasificación de Keros para la configuración del techo etmoidal (tipo I de 1 a $3 \mathrm{~mm}$, tipo II de 4 a $8 \mathrm{~mm}$ o tipo III de 8 a 16 $\mathrm{mm}$, medido entre la lámina cribosa y el techo etmoidal anterior).

- Tabique nasal: desviación y presencia de espolón.

Todas las variantes anatómicas que pueden existir en forma bilateral fueron evaluadas por separado para ambos lados.

\section{Resultados}

Descripción general de la muestra:

El rango de edad fue de 18 a 95 años, con un promedio de 42 años ( $\mathrm{DE}+/-17)$. De los 100 pacientes 56 fueron mujeres y 44 fueron hombres.

\section{Proceso uncinado}

De los 200 procesos uncinados estudiados un $68 \%$ presentó inserción superior en la lámina papirácea, $24 \%$ en la lámina cribosa y $8 \%$ en el cornete medio, sin diferencias significativas según lateralidad. En dos casos se pesquisó bulla uncinada, siendo bilateral en uno de ellos. 


\section{Cornetes}

En relación a la configuración y neumatización del cornete medio, de los 200 evaluados, un $49 \%$ fue normal (sin configuración paradojal ni neumatización); el 24\% tuvo configuración paradojal; un $14 \%$ tuvo configuración normal con neumatización parcial; el $8 \%$ tuvo configuración paradojal más neumatización parcial; y un 5\% correspondió a concha bullosa (Figura 1), sin diferencias significativas según lateralidad. No se registraron casos de cornete medio secundario. En 9 pacientes se evidenció la presencia de concha suprema, siendo en 7 de ellos bilateral (Figura 2).

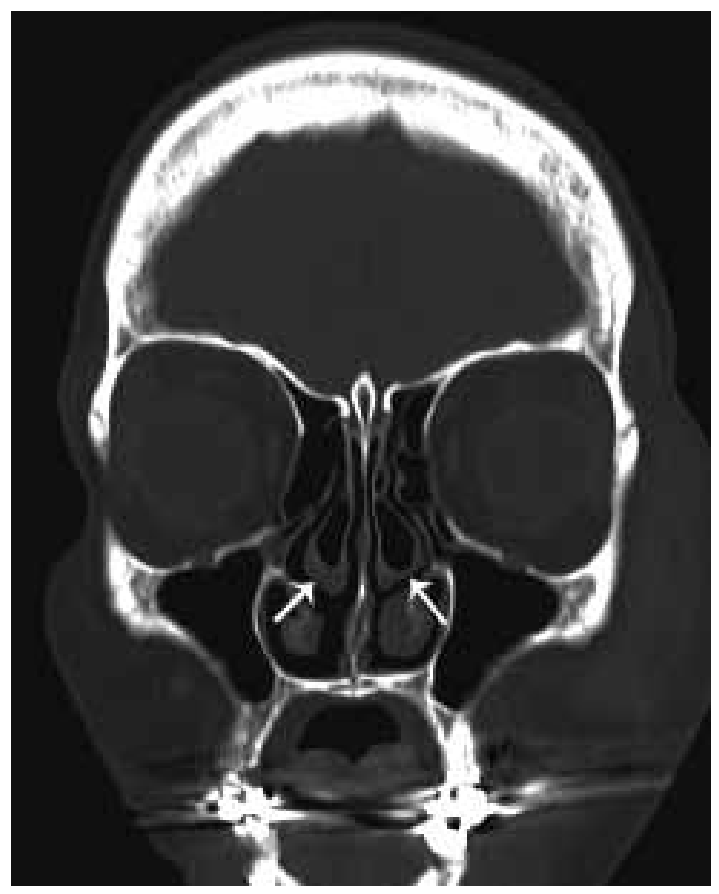

Figura 1. Concha bullosa bilateral.

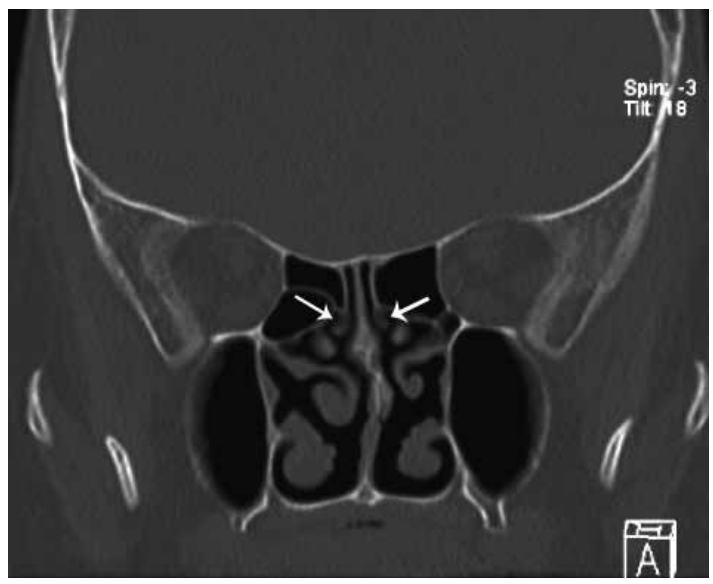

Figura 2. Concha suprema bilateral.

\section{Seno frontal}

De los 200 senos frontales estudiados un $66 \%$ drenaba al meato medio y un $32 \%$ al infundíbulo etmoidal, sin diferencias significativas según lateralidad. En el 2\% restante existía hipoplasia del seno frontal, sin poder definirse el drenaje de éste. Según la clasificación de Kuhn del receso y seno frontal, un $56 \%$ correspondió a tipo 1 (pequeña celdilla sobre Agger Nasi); $22 \%$ a tipo 2 (varias celdillas sobre Agger Nasi); $8 \%$ a tipo 3 (gran celda sobre Agger Nasi con extensión al seno frontal); y $7 \%$ a tipo 4 (celdilla aislada en el seno frontal), sin diferencias significativas según lateralidad (Figura 3). En el 7\% restante no se observó celdilla sobre Agger Nasi.
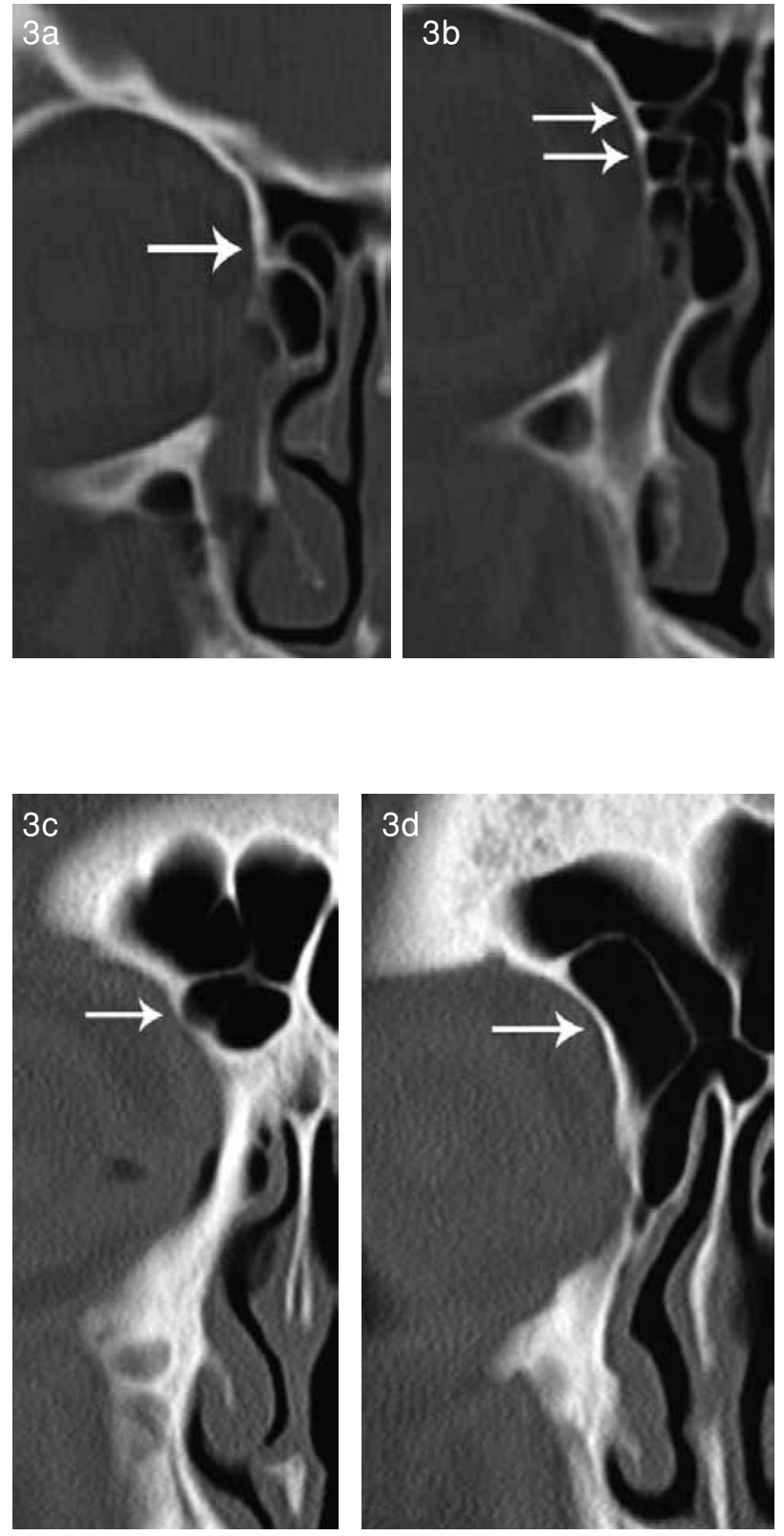

Figura 3. Clasificación de Kuhn del receso y seno frontal A: Kuhn tipo 1; B: Kuhn tipo 2; C: Kuhn tipo 3; D: Kuhn tipo 4. 


\section{Celdillas etmoidales}

El número promedio de celdillas etmoidales en las $100 \mathrm{TC}$ estudiadas fue de $7(\mathrm{DE}+/-1)$, tanto a derecha como izquierda. De las 100 TC, en un $66 \%$ se observó eminencia nasal neumatizada (Agger Nasi), la cual fue bilateral en el $82 \%$ de éstos; en un $24 \%$ celdilla infraorbitaria (Haller) (Figura 4), la cual fue bilateral en un $46 \%$ de éstos; y en un $25 \%$ celdilla esfenoetmoidal (Onodi) (Figura 5), la cual fue bilateral en un $56 \%$ de éstos.

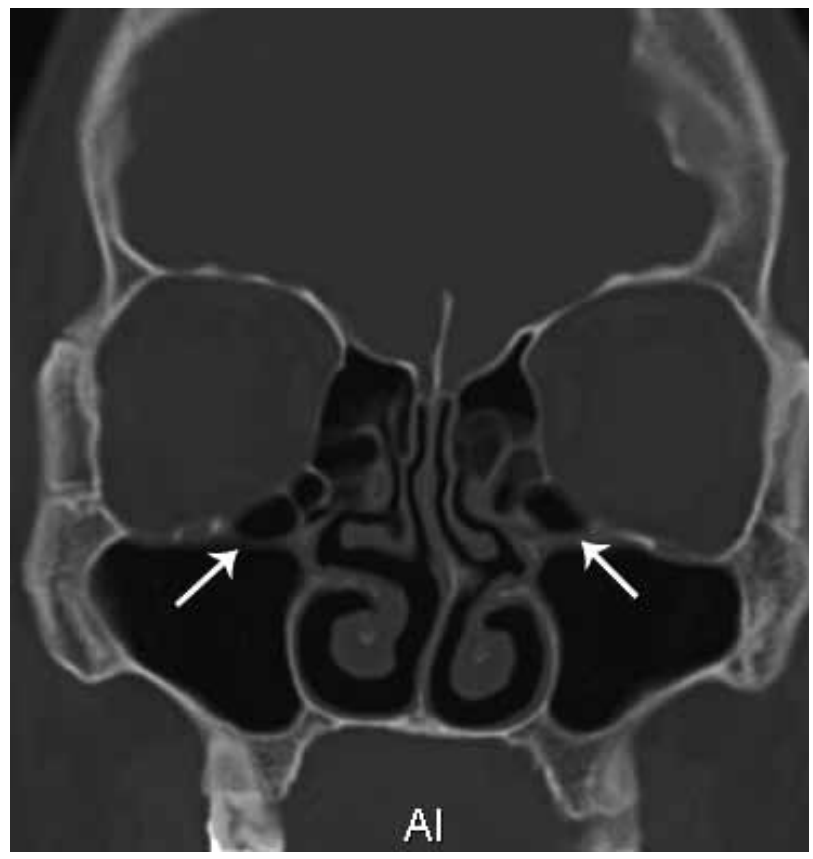

Figura 4. Celdilla infraorbitaria (Haller) bilateral.

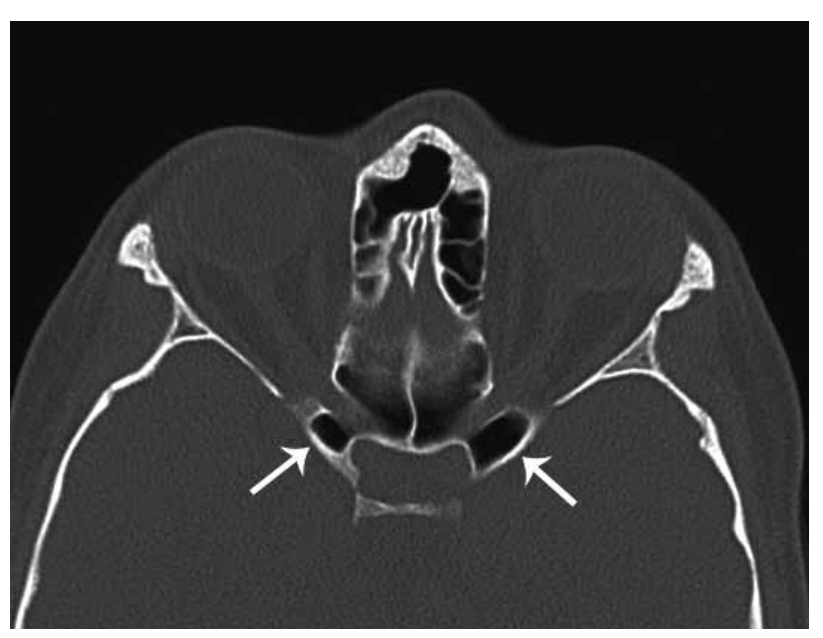

Figura 5. Celdilla esfenoetmoidal (Onodi) bilateral.

\section{Seno maxilar}

Se registró hipoplasia unilateral del seno maxilar en 3 pacientes (Figura 6), no se registraron casos de agenesia.

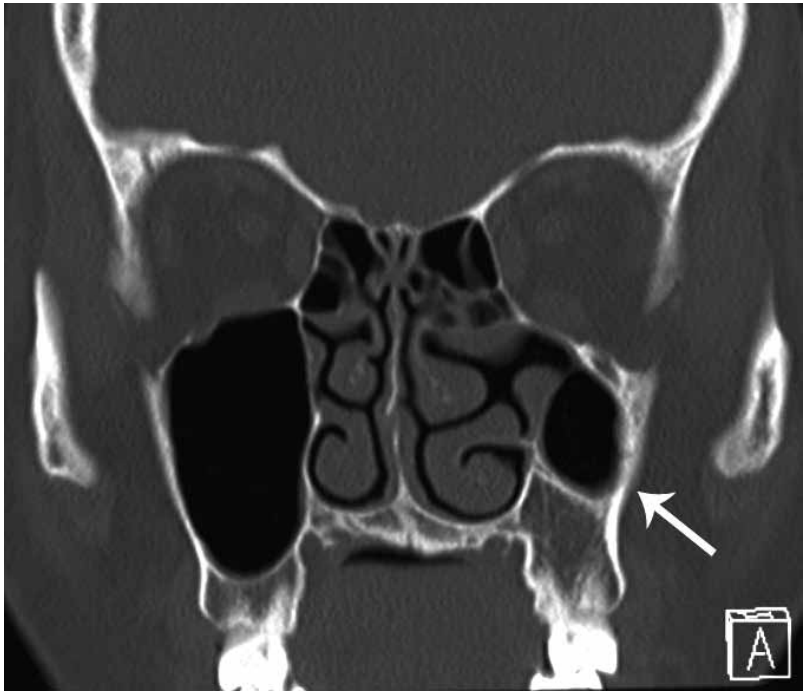

Figura 6. Hipoplasia del seno maxilar izquierdo.

\section{Seno esfenoidal}

De las 100 TC, un $21 \%$ presentó más de un tabique intraesfenoidal y un $43 \%$ presentó inserción del tabique intraesfenoidal en relación al canal carotídeo (Figura 7). En 5 casos se registró dehiscencia de la pared ósea en relación al canal carotídeo (Figura 6), siendo uno de éstos bilateral. Según la clasificación de Hamberger para neumatización del seno esfenoidal, un $80 \%$ presentó configuración selar, un $19 \%$ configuración preselar y un 1\% configuración conchal (Figura 8).

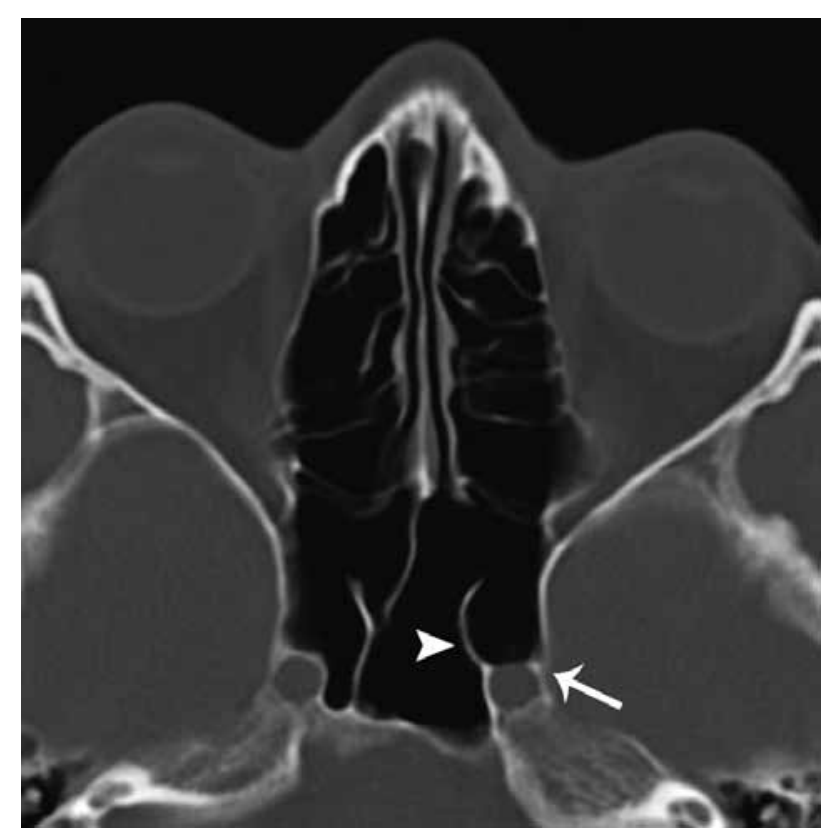

Figura 7. Dehiscencia de la pared ósea e inserción de tabique intraesfenoidal en relación al canal carotídeo flecha: dehiscencia de la pared ósea en relación al canal carotídeo; cabeza de flecha: inserción de tabique intraesfenoidal en al canal carotídeo. 

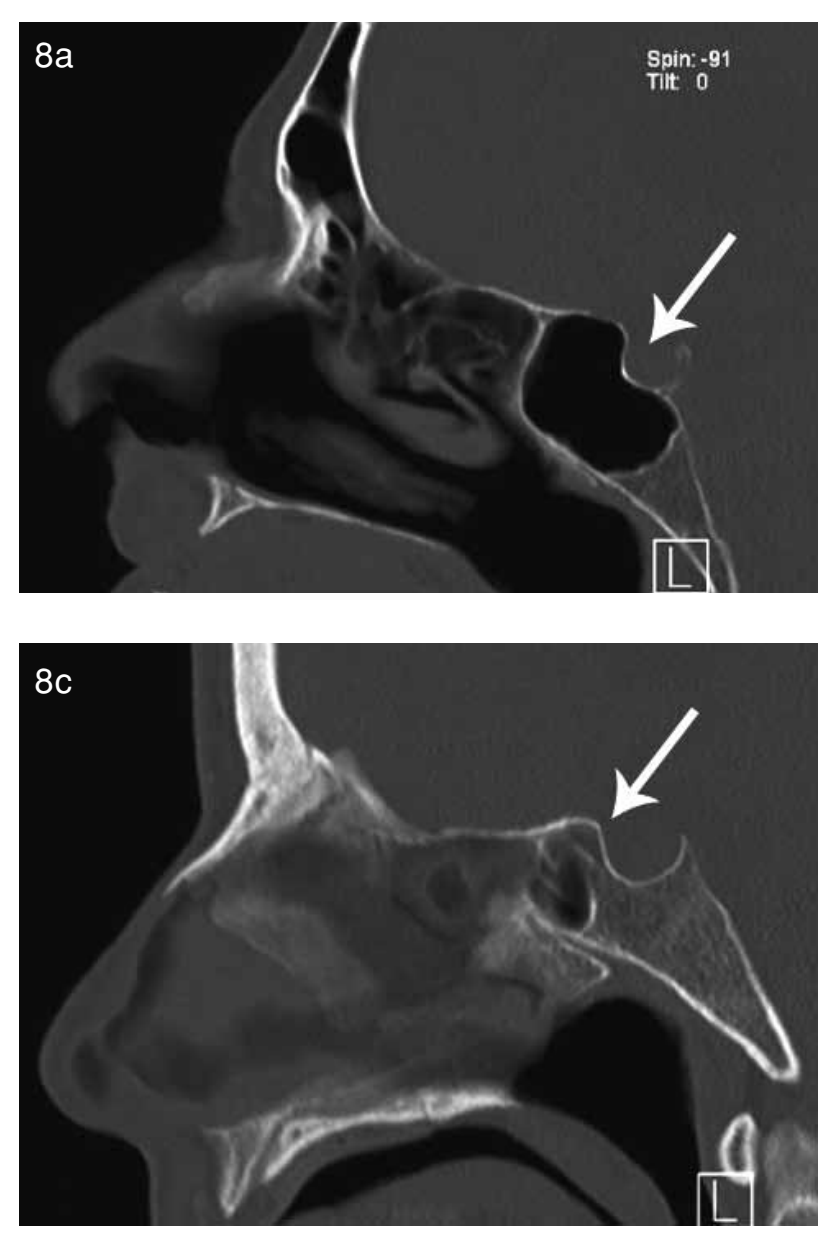

\section{Fosa olfatori}

De las 200 fosas olfatorias evaluadas, según la clasificación de Keros de la configuración del techo etmoidal, se evidenció un $2 \%$ de casos con configuración tipo I ( 1 a $3 \mathrm{~mm}$ ), un $28 \%$ con configuración tipo II (4 a $8 \mathrm{~mm}$ ) y un $70 \%$ con configuración tipo III (8 a $16 \mathrm{~mm}$ ) (Figura 9).

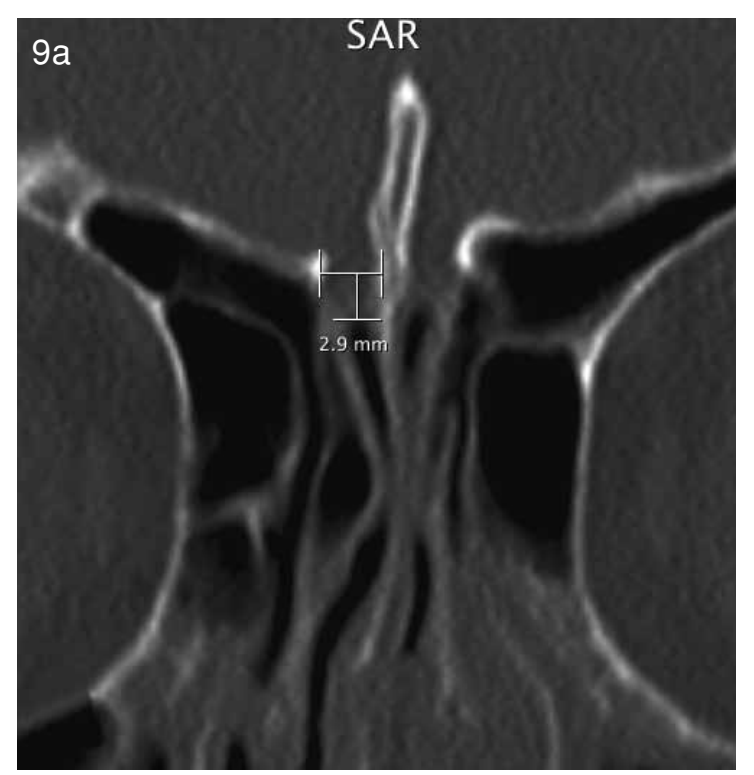

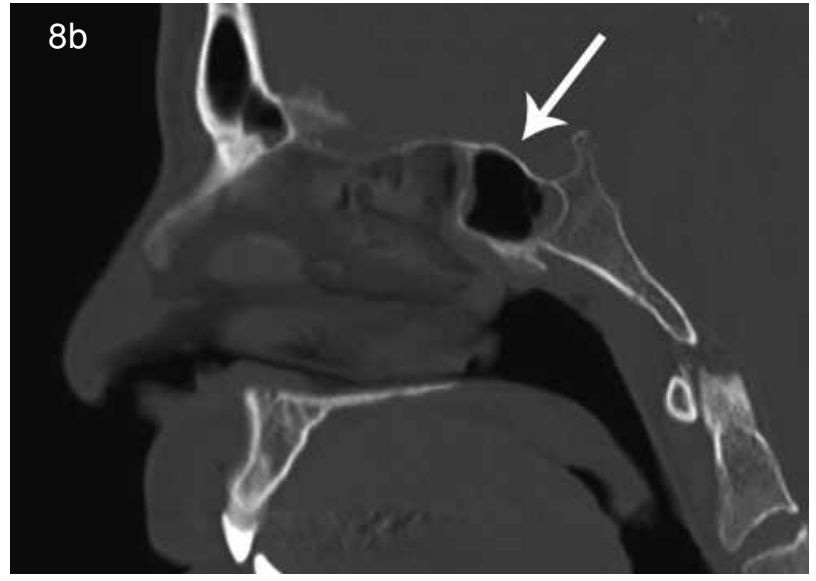

Figura 8. Clasificación de Hamberger para neumatización del seno esfenoidal. a) configuración selar. b)configuración preselar. c) configuración conchal.
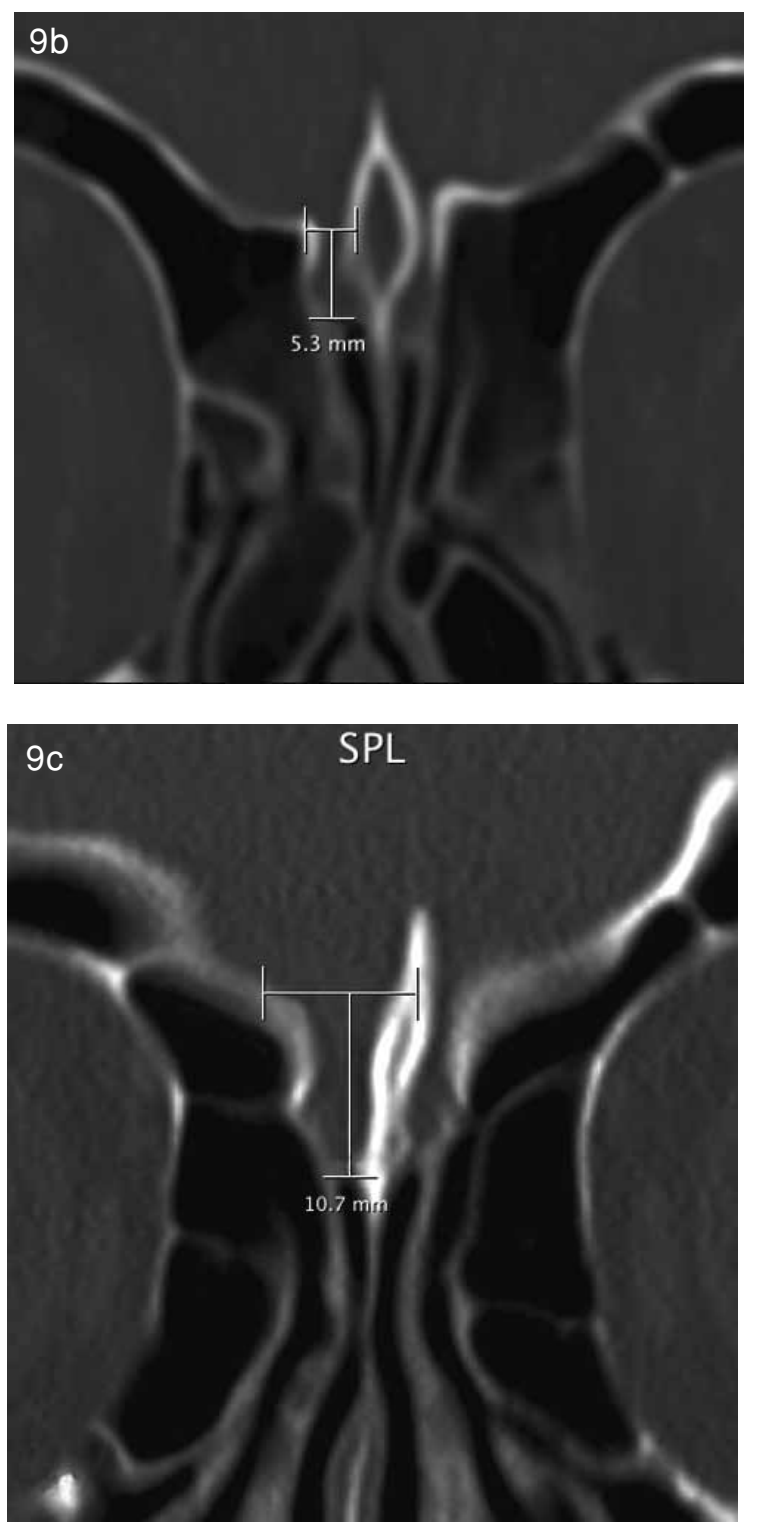

Figura 9. Clasificación de Keros de la fosa olfatoria. a) Keros tipo I. b) Keros tipo II. c) Keros tipo III. 


\section{Tabique nasal}

De las 100 TC evaluadas el $83 \%$ presentó desviación del tabique nasal, sin diferencia significativa entre septodesviación a derecha o a izquierda. En 7 de estos casos existía una configuración en "S itálica". El espolón del tabique estuvo presente en un $45 \%$ de los casos, sin diferencia significativa entre espolón a derecha o a izquierda.

Todos los valores numéricos de las variantes anatómicas naso-sinusales descritas en los párrafos previos se resumen en la tabla I.

Tabla I. Variantes anatómicas naso-sinusales

Variante

Proceso unciforme

Inserción superior en lámina papirácea

Inserción superior en lámina cribosa

Inserción superior en cornete medio

Bulla uncinada

\section{Cornetes}

Cornete medio paradojal

Cornete medio con neumatización parcial

Cornete medio paradojal con neumatización parcial

Concha bullosa

Cornete medio secundario

Concha suprema
Porcentaje

$$
\begin{array}{r}
68 \% \\
24 \% \\
8 \% \\
2 \%
\end{array}
$$

$24 \%$

$14 \%$

$8 \%$

$5 \%$

$0 \%$

$9 \%$

$2 \%$

$66 \%$

$32 \%$

$56,22,8$ y $7 \%$
$66 \%$

$24 \%$

$25 \%$

Celdilla infraorbitaria (Haller)
Celdilla esfenoetmoidal (Onodi)

Eminencia nasal neumatizada (Agger Nasi)

Seno maxilar

Hipoplasia

Seno esfenoidal

Más de un tabique intraesfenoidal

Inserción de tabique intraesfenoidal en el CC

Dehiscencia de la pared ósea en relación al CC

$5 \%$

Clasificación de Hamberger: selar, preselar y conchal

80,19 y $1 \%$

Fosa olfatoria

Clasificación de Keros: tipo I, tipo II y tipo III

2,28 y $70 \%$

Tabique nasal

Desviación

$83 \%$

Espolón $\quad 45 \%$

CC: canal carotídeo

Muestra compuesta por 100 pacientes 


\section{Discusión}

\section{Proceso uncinado}

El conocimiento del sitio de inserción superior del proceso uncinado es fundamental en la CEF de cavidades paranasales. Una fractura inapropiada de éste puede llevar a lesión de la lámina papirácea con invasión de la órbita o lesión de la lámina cribosa con trastorno olfatorio y fístula de líquido cefalorraquídeo, según donde éste se inserte ${ }^{(2)}$. En nuestro estudio, el sitio de inserción superior más frecuente fue la lámina papirácea con un $68 \%$, lo cual es similar a lo descrito por Liu y cols., quienes describen $70 \%$ de inserción superior del proceso en la lámina papirácea ${ }^{(7)}$. Nuestros resultados arrojaron sólo un $2 \%$ de bulla uncinada, lo cual coincide con lo descrito en la literatura nacional ${ }^{(8)}$ e internacional(9) , que describe a esta variante como una entidad poco frecuente, que puede determinar obstrucción del complejo ostio-meatal (COM). Por los motivos antes mencionados, resulta importante describir en el informe radiológico el sitio de inserción superior y la presencia de neumatización del proceso uncinado.

\section{Cornetes}

Se define como verdadera concha bullosa a la neumatización de la lámina vertical y bulbo inferior del cornete medio, hallazgo que estuvo en el $5 \%$ de los casos en nuestro estudio. Este valor se sitúa por debajo de lo descrito en la literatura nacional ${ }^{(8)}$ e internacional ${ }^{(10)}$, que en muchos de ellos considera dentro de esta cifra también a los casos de neumatización parcial. La configuración paradojal del cornete medio estuvo presente en el $24 \%$ de los casos en nuestro estudio; cifra que varía entre 3 y $27 \%$ en lo descrito en la literatura ${ }^{(10,11)}$. Ambas variantes anatómicas pueden contribuir a la obstrucción del COM y favorecer el desarrollo de sinusitis aguda recurrente y/o crónica. La presencia de concha suprema, presente en 9 casos de nuestro estudio, puede ser confundida con un pólipo u osteoma si no se dispone de estudio con TC, particularmente cuando es unilateral.

\section{Seno frontal}

El drenaje del seno frontal ocurre mayoritariamente, en $2 / 3$ de los casos, hacia el meato medio. Esto se encuentra determinado por la inserción superior del proceso uncinado, que mayoritariamente es en la lámina papirácea. Cuando el proceso uncinado se inserta en la lámina cribosa o en el cornete medio, el drenaje del seno frontal es hacia el infundíbulo etmoidal. La clasificación de Kuhn del receso y seno frontal arrojó que los tipos I y II (celdillas localizadas en el área del receso frontal) son los más frecuentes, con un 56 y $22 \%$ respectivamente. Esto resulta similar a lo descrito por Kuhn y cols., quienes mencionan un 37 y $19 \%$ respectivamente ${ }^{(12)}$. Está demostrado en la literatura que pacientes con configuración de Kuhn tipo III y IV (celdillas que invaden el seno frontal) tienen mayor incidencia de sinusitis frontal e hiperneumatización del seno frontal(13). Estos hallazgos son fácilmente identificables en los estudios de TC y debiesen consignarse en el informe, dada su relación con patología del seno frontal.

\section{Celdillas etmoidales}

El número promedio de celdillas etmoidales en las 100 TC estudiadas fue de 7 (DE+/-1), cifra similar a la descrita en textos clásicos de anatomía ${ }^{(14)}$. Respecto a las principales celdillas accesorias existentes en esta región, la celdilla Agger Nasi (eminencia nasal neumatizada) estuvo presente en el $66 \%$ de la muestra, hallazgo frecuente según la literatura nacional ${ }^{(8)} \mathrm{e}$ internacional( ${ }^{(1)}$, que debiese considerarse como una "constante anatómica" más que una "variante anatómica"; la presencia de celdilla Agger Nasi de gran tamaño podría relacionarse con sinusitis crónica frontal, al impedir el drenaje del receso frontal. La celdilla de Haller (celdilla infraorbitaria) estuvo presente en un $24 \%$ de la muestra, mayormente unilateral, cifra similar a la descrita en la literatura internacional ${ }^{(1)} y$ algo mayor que la literatura nacional ${ }^{(8)}$. Esta celdilla es un reparo anatómico de importancia, pues está implicada en el desarrollo de sinusitis maxilar unilateral recurrente y su persistencia en el postoperatorio de CEF es indicador de fracaso de la antrostomía maxilar ${ }^{(1,8)}$. Finalmente, la celdilla de Onodi (celdilla esfenoetmoidal), en estrecha relación al nervio óptico, estuvo presente en un $25 \%$ de la muestra, cifra levemente mayor a la descrita en estudios de anatomía radiológica nacional $(13 \%)^{(8)}$ e internacional $(8-14 \%)^{(1)}$, pero menor que la cifra descrita en estudios con cadáveres $(60 \%)^{(15)}$. Es fundamental identificar esta celdilla y mencionarla en el informe radiológico del estudio preoperatorio de cirugía endoscópica al abordar esfenoides y etmoides posterior, tanto para enfrentar patología de la región como para utilizar estas cavidades como vía de paso en la resección de tumores selares, debido a la posibilidad de lesión accidental del nervio óptico.

\section{Seno maxilar}

Se registró hipoplasia unilateral del seno maxilar en 3 pacientes, hallazgo poco frecuente, similar a lo descrito en la literatura nacional(8) e internacional ${ }^{(1,11)}$. Esta variante del desarrollo puede ser mal diagnosticada como sinusitis maxilar crónica ${ }^{(1)}$, por lo tanto, resulta importante su adecuado reconocimiento e informe por parte de los radiólogos.

\section{Seno esfenoidal}

Las variantes anatómicas del seno esfenoidal resultan importantes, tanto para la CEF de cavidades paranasales como para la cirugía transesfenoidal de la región hipofisiaria. De particular importancia es la dehiscencia de la pared ósea en relación al canal carotídeo, hallazgo encontrado en el $5 \%$ de la muestra, similar a lo descrito en la literatura ${ }^{(1)}$. Si el cirujano no está al tanto de esta variante anatómica puede producir lesión arterial de difícil manejo, eventualmente fatal. La inserción del tabique 
intraesfenoidal, presente en el $43 \%$ de nuestra muestra es otro factor que puede asociarse a lesión arterial, al fracturar inapropiadamente este tabique durante la cirugía. El mapeo de tabicación del seno aportado por la TC es de gran utilidad y debe ser mencionado en el informe radiológico, pues permite realizar abordajes transesfenoidales seguros, disminuyendo el tiempo operatorio y eventuales complicaciones. Finalmente, el patrón de neumatización del seno esfenoidal es otro factor que el cirujano debe considerar para un abordaje transesfenoidal de la región hipofisiaria; según la clasificación de Hamberger, en nuestra muestra, un $80 \%$ presentó configuración selar, un $19 \%$ configuración preselar y un 1\% configuración conchal, cifras similares a las descritas en la literatura ${ }^{(16)}$. El conocimiento preoperatorio de la configuración del seno, fácilmente visualizada en reconstrucciones sagitales de TC, permitirá una adecuada programación de la cirugía, siendo en ocasiones necesaria instrumentación de uso no habitual o sistemas de navegación.

\section{Fosa olfatoria}

La configuración de la fosa olfatoria es un factor importante para el cirujano. La literatura señala que techos etmoidales bajos presentan menor riesgo de fístula de líquido cefalorraquídeo durante la cirugía( ${ }^{(8)}$. En nuestro estudio, se evidenció un $2 \%$ de configuración tipo I (1 a $3 \mathrm{~mm}$ ); $28 \%$ de configuración tipo II ( 4 a $8 \mathrm{~mm}$ ); y $70 \%$ de configuración tipo III (8 a $16 \mathrm{~mm}$ ). Estas cifras muestran un franco predominio de techos etmoidales altos. La literatura internacional, realizada fundamentalmente en población caucásica-europea, señala como más frecuentes los techos etmoidales de altura media y alta (Keros tipo II y III) (10,17); el único estudio nacional disponible señala como más frecuentes los techos etmoidales bajos (Keros tipo I) ${ }^{(8)}$. Posiblemente se necesita de estudios con mayor cantidad de pacientes y con consideración del factor étnico para definir cuál es el patrón de configuración de la fosa olfatoria en población chilena.

\section{Tabique nasal}

Dos de las variantes anatómicas más frecuentes de la región naso-sinusal se sitúan en el tabique nasal, correspondiendo a la septodesviación y el espolón del tabique, presentes en un 83 y $45 \%$ de nuestra muestra respectivamente. La literatura nacional 8 e internacional describe cifras similares ${ }^{(11)}$.

Posiblemente resulte conveniente realizar evaluaciones semejantes con un mayor número de casos, con el objetivo de describir en una muestra representativa del país la prevalencia de las variantes anatómicas de la región naso-sinusal.

\section{Conclusión}

Describimos la prevalencia de las principales variantes anatómicas de la región naso-sinusal en una muestra de población chilena, mediante el uso de TC, demostrando en general correlación con lo descrito en la literatura nacional e internacional. El médico radiólogo debe saber reconocer estas variantes, valorar su importancia y mencionarlas en su informe radiológico.

\section{Bibliografía}

1. Kantarci M, Karasen RM, Alper F, Onbas O, Okur A, Karaman $A$. Remarkable anatomic variations in paranasal sinus region and their clinical importance. Eur $\mathrm{J}$ Radiol 2004; 50: 296-302.

2. Bayram M, Sirikci A, Bayazit YA. Important anatomic variations of the sinonasal anatomy in light of endoscopic surgery: a pictorial review. Eur Radiol 2001; 11: 1991-1997.

3. Fatterpeaker GM, Delman BN, Som PM. Imaging the paranasal sinuses: Where we are and where we are going. Anat Rec (Hoboken) 2008; 291(11): 1564-1572.

4. Zinreich SJ. Progress in sinonasal imaging. Ann Otol Rhinol Laryngol Suppl 2006; 196: 61-65.

5. Clemente MP. Surgical anatomy of the paranasal sinus. In: Levine $\mathrm{H}$, Clemente MP, editors. Sinus surgery: endoscopic and microscopic approaches. 1st Ed. Thieme 2004. Chapter 1: 1-56.

6. Wormald PJ. Anatomy of the frontal recess and frontal sinus with tri-dimentional reconstruction. In: Wormald PJ, editor. Endoscopic sinus surgery: anatomy, threedimentional reconstruction, and surgical technique. 2nd Ed. Thieme 2008. Chapter 6: 43-81.

7. Liu SC, Wang $\mathrm{CH}$, Wang HW. Prevalence of the uncinate process, agger nasi cell and their relationship in a Taiwanese population. Rhinology 2010; 48(2): 239-244.

8. Cabezón R, Valdés R, Breinbauer H, Ramírez C, Grau C, Iñíguez $\mathrm{R}$. Variantes anatómicas relevantes en tomografía computarizada de cavidades perinasales. Rev Otorrinolaringol Cir Cabeza Cuello 2010; 70(3): 223-230.

9. Bolger W. Paranasal sinus bony anatomic variations and mucosal abnormalities: CT analysis for endoscopic sinus surgery. Laryngoscope 1991; 101(1): 56-64.

10. Arslan H, Aydinlio lu A, Bozkurt M, Egeli E. Anatomic variations of the paranasal sinuses: CT examination for endoscopic sinus surgery. Auris Nasus Larynx 1999; 26(1): 39-48.

11. Pérez-Piñas I, Sabaté J, Carmona A, Catalina-Herrera CJ, Jiménez Castellanos J. Anatomical variations in the human paranasal sinus region studied by CT. J Anat 2000; 197(2): 221-227.

12. Lee WT, Kuhn FA, Citardi MJ. 3D computed tomographic analysis of frontal recess anatomy in patients without frontal sinusitis. Otolaryngol Head Neck Surg 2004; 131(3): 164-173.

13. Yildirim A. Is it more reasonable to categorize frontal cells on the basis of their location rather than on their type? Ear Nose Throat J 2010; 89(9): 19-21.

14. Rouvière H, Delmas A. Senos paranasales o cavidades neumáticas anexas a las cavidades nasales. En: Anatomía humana: descriptiva, topográfica y funcional. $10^{\mathrm{a}} \mathrm{Ed}$. Masson 1999. Tomo I: 298: 302.

15. Thanaviratananich $\mathrm{S}$, Chaisiwamongkol K, Kraitrakul S, Tangsawad W. The prevalence of Onodi cell in adult Thai cadavers. Ear Nose Throat J 2003; 82(3): 200-204.

16. Hamid O, El Fiky L, Hassan O, Kotb A, El Fiky S. Anatomic variations of the sphenoid sinus and their impact on trans-sphenoid pituitary surgery. Skull Base 2008; 18(1): 9-15.

17. Erdem G, Erdem T, Cem Miman M, Ozturan O. A radiological anatomic study of the cribiform plate compared with constant structures. Rhinology 2004; 42(4): 225-229. 